\title{
Neuroprotection with Glycine-2-Methylproline-Glutamate (G-2MePE) after hypoxic-ischemic brain injury in adult rats
}

December 9, $2014 \cdot$ Volume $6 \cdot$ Original Research

Sam Mathai $\mathrm{PhD}^{1}$, Paul W. Harris $\mathrm{PhD}^{2}$, Margaret A. Brimble $\mathrm{PhD}^{3}$, Alistair J. Gunn PhD ${ }^{4}$, Jian Guan $\mathrm{PhD}^{5}$

1 Department of Department of Physiology, Faculty of Medicine and Health Science, The University of Auckland, The Liggins Institute, , , New Zealand

2 Department of School of Chemical and Biological Sciences, The University of Auckland, , , New Zealand

3 Department of School of Chemical and Biological Sciences, Faculty of Medicine and Health Science, The University of Auckland, The Centre for Brain Research, , , New Zealand

4 Department of Department of Physiology, Faculty of Medicine and Health Science, The University of Auckland, The Centre for Brain Research, , , New Zealand

5 Department of The Centre for Brain Research, Faculty of Medicine and Health Science, The Liggins Institute, the University of Auckland, , , New Zealand

Article citation: Mathai S, Harris PW, Brimble MA, Gunn AJ, Guan J. Neuroprotection with Glycine-2-Methylproline-Glutamate (G-2MePE) after hypoxic-ischemic brain injury in adult rats: Neuroprotection of G-2MePG. J Exp Stroke Transl Med 2014 Dec 9, Volume 6. 1-31. Online access at http://www.aestm.com/article/neuroprotection-with-glycine-2-methylproline-glutamate-g-2mepe-after-hypoxic-ischemic-brain-injury-in-adult-rats/

Correspondence should be sent to:Dr Jian Guan, the Liggins Institute, the University of Auckland, Private Bag 92019, Auckland, New Zealand Phone: +64-9-9236134 Fax: +64-9-3737497 Email: j.guan@auckland.ac.nz

\begin{abstract}
Background and Purpose: Hypoxic-ischemic brain injury, due to reduced supply of oxygen to brain, is a major cause of death and disability. There is no exclusive treatment available so far. Glycine-2-Methylproline-Glutamate (G-2MePE, NNZ 2566), an analogue of Glycine-Proline-Glutamate reduces neuronal injury after focal ischemia in adult rats. The current study investigated into the neuroprotective effects of G-2MePE after global hypoxic-ischemic brain injury in adult rats.Methods: Adult male rats received a single sub cutaneous injection of G-2MePE $(1.2 \mathrm{mg} / \mathrm{kg}) 3 \mathrm{~h}$ post hypoxic-ischemic brain injury or the same volume of normal saline. Brains were extracted 5 days after the treatment. Tissue damage in the cortex, hippocampus and striatum was assessed. Neuronal survival, glial reactions, caspase- 3 activity and TNF- $\alpha$ cytokine activity were also assessed.Results: The treatment with G-2MePE was associated with a significant reduction of tissue damage, improvement in neuronal survival, reduction in reactive microglia, TNF- $\alpha$ positive cells and caspase-3 positive cells in hippocampus and cortex but an elevation of astrocytosis.Conclusions: Neuroprotection with G-2MePE after hypoxic-ischemic brain injury in adult rats is associated with reduced neuronal necrosis, apoptosis, modulated inflammatory responses and augmented astrocytosis.
\end{abstract}

\section{Introduction}

Stroke is a leading cause of death and disability in developed countries affecting 1 in 250 persons. Ischemic stroke is the most common type which occurs when blood supply to the brain is reduced, causing neurons starving for oxygen, resulting in neuronal death or dysfunction, culminating in corresponding loss of neurologic function (Dirnagl et al. , 1999, Chan, 2001, Hayashi and Abe, 2004). A range of promising drugs have been tested in vitro and in vivo in the past; but the majority have failed to translate to the clinic (Gleichmann et al. , 2000, Kawano et al. , 2001, Domanska-Janik et al. , 2004, Mohammad et al. , 2004, Wahlgren and Ahmed, 2004, Kilic et al. , 2006, Switzer and Hess, 2006). One obvious reason is the lack of animal models that represent the pathogenesis and pathology of human stroke. Thus testing the potential compound using multiple animal models may provide a more objective view for their preclinical development. The only therapy with proven benefits for acute ischemic stroke patients is the intravenous thrombolytic therapy with recombinant tissue-type plasminogen activator (rt-PA) within $3 \mathrm{~h}$ of the onset of stroke (Fisher and Schaebitz, 2000, Acampa et al. , 2014), the window which has been recognised as critical for preventing neuronal death after stroke.

N-terminal glycine-proline-glutamate (GPE) is a neurobioactive tri peptide, naturally cleaved from IGF-1 by an acid protease (Sara et al. , 1989). GPE crosses the blood brain barrier (BBB) and can prevent brain injury after embolic-stroke in aged rats (Guan et al., 2004, Shapira et al. , 2009) and after hypoxic-ischemic (HI) injury in developing adult brain (Sizonenko et al. , 2001). GPE is small and does not activate the IGF-1 receptor (Alonso De Diego et al. , 2006). GPE has a short half-life $(<2 \mathrm{~min})$ in plasma due to a rapid peptidase-mediated breakdown (Batchelor et al. , 2003).

G-2MePE is a structural analogue of GPE designed to improve protease resistance by alpha methylation of the proline moiety (Harris and Brimble, 2006). It is known that G-2MePE can reduce injury after focal brain injury and trauma (Bickerdike et al. , 2009) and HI injury in neonatal rat (Svedin et al. , 2007). Reduced inflammation has been reported among the protective effects of G-2MePE (Svedin et al. , 2007, Lu et al. , 2009, Wei et al. 2009, Cartagena et al. , 2013). There are various rodent models of stroke designed for testing the efficacy of neuroprotectants for clinical use with some of them reproducing the pathological changes and others mimicking the pathophysiological events of human stroke. Focal injury models are often severe with limited windows of opportunity to intervene and short outcome evaluation at 24h (Zhou et al. , 2013, Gheibi et al. , 2014). Therefore testing G-2MePE in different animal models of focal brain injury is desirable. HI induces focal brain damage through a global hypoxic insult with local reduction in blood flow. This approach allows progressive brain damage and provides the opportunity to evaluate longer term outcome. Thus the aim of the study was to examine the treatment effects of G-2MePE after HI brain injury in adult rats.

\section{Material and MethodsAnimals}

All experimental protocols in this study were approved by the Auckland University Ethics committee. Adult male Wistar rats (280-310g) were 

with $60 \%$ relative humidity and free access to food and water ad libitum.

\section{HI brain injury}

The procedure has been previously described (Guan et al. , 2004). Animals were anesthetised with $3 \%$ isoflurane/oxygen and the right common carotid artery was permanently ligated with $0 / 3$ silk thread. Rats were left at room temperature for $2 \mathrm{~h}$ and then placed in an incubator at $90 \pm 5 \%$ relative humidity and temperature $31 \pm 0.5^{\circ} \mathrm{C}$ for $30 \mathrm{~min}$. Hypoxia was induced by quickly reducing the oxygen level to $6 \%$ by introducing nitrogen gas and then maintained at $6 \% \pm 0.2 \%$ level by adjusting the flow of nitrogen and air. Hypoxia continued for 15 min under close monitoring.

\section{Drug preparation and administration}

G-2MePE (Neuren Pharmaceuticals, Auckland) was dissolved in $0.9 \%$ saline solution. The experimenter was masked to the treatment groups throughout the experiments. Rats were randomly divided into two treatment groups for receiving either vehicle (0.3 $\mathrm{ml}$ saline, $\mathrm{n}=20)$ or an equal volume of G-2MePE dissolved in saline $(1.2 \mathrm{mg} / \mathrm{kg}, \mathrm{n}=18)$ subcutaneously $(\mathrm{SC})$, $3 \mathrm{~h}$ after the HI. After the SC injection, animals were housed in groups of 3 in the holding room and weighed daily to ensure their well-being.

\section{Brain histological preparation}

Five days after the experiments rats were euthanized by an intraperitoneal injection of pentobarbital (125mg/kg) and the brains were transcardially perfused with normal saline followed by fixation with $10 \%$ neutral buffered formalin, by gravity feed. The brains were left in formalin solution for 2 days for continued fixation, coronally sliced to $3 \mathrm{~mm}$ blocks, processed and paraffin embedded. $8 \mu \mathrm{m}$ thick coronal sections were cut at 3 levels i.e. at the level of striatum, at the level of cerebral cortex \& dorsal hippocampus and at the level of cortex \& ventral horn of the hippocampus (Guan et al. , 2000, Shapira et al. , 2009) and mounted on albumen-chrome alum pre-coated slides.

\section{Brain damage assessment}

The degree of tissue damage was assessed using a scoring system after thionin-acid fuchsine staining. Briefly, deparaffinised and hydrated sections were stained with aqueous solutions of thionin and acid fuchsine. Three coronal sections from each brain region were used for scoring in the ipsilateral (ischemic) hemisphere. Tissue damage was assessed at three levels anterior from inter-aural reference zero plane, (A-P 6 , 4.5 and $4.2 \mathrm{~mm}$ ) for the lateral cortex; at two levels (A-P 4.5 and $4.2 \mathrm{~mm}$ ) for the hippocampus \& the dentate gyrus and at one level (A-P $6 \mathrm{~mm})$ for the striatum respectively (Paxinos and Watson, 1982). A 4 point scoring system was used to assess the histopathological damage $(0=$ no damage; 1 = few cells damaged; $2=<$ half of brain tissue damaged; $3=>$ half of the tissue damaged and $4=$ almost all neurons died and no tissue survived) (Rochester et al. , 2005). Brain tissue with selective neuronal death, pan-necrosis and cellular reaction was treated as damaged (Guan et al. , 2000). Neuronal death was confirmed by the presence of acidophilic (pink) cytoplasm and contracted nuclei (Brown and Brierley, 1972, Auer et al. , 1985, Guan et al. , 1993). Scoring was done by an experimenter masked to the study. Average tissue damage scores in different regions were statistically analysed.

\section{Immunohistochemical labelling}

The extent of neuronal survival in different parts of the brain, caspase- 3 expression and inflammatory responses in the cortex \& hippocampus were evaluated by immunohistochemical staining in brain paraffin preparations from experiment 1.10 sections each from the experimental groups were taken for each antibody labelling. Primary and secondary antibodies used and their dilutions were as follows. (i) monoclonal mouse anti-NeuN for labelling surviving neurons (Chemicon International, Temecula, CA, USA, 1:200) (ii) goat polyclonal anti-ionised calcium-binding adaptor molecule1(lba-1) for reactive microglia (Abcam, via Sapphire Bioscience, Hamilton, New Zealand , 1:200) (iii) polyclonal rabbit anti-glial fibrillary acidic protein (GFAP) for reactive astrocytes (Chemicon International, Temecula, CA, USA, 1:500), (iv) monoclonal mouse anti-TNF- $\alpha$ for proinflammatory cytokine TNF- $\alpha$ (AbD Serotec, Auckland, 1:200) and (v) rabbit polyclonal anti-cleaved caspase-3 ASP 175 for cells undergoing caspase-3 mediated apoptosis (Cell Signalling Technology, U.K; 1:200) (vi) biotin conjugated anti-mouse, anti-goat and anti-rabbit secondary bodies (Vector Laboratories, Burlingame, CA, 1:200)

Xylene-deparaffinised slides were hydrated in decreasing concentrations of ethanol and washed with $0.1 \mathrm{~m} / \mathrm{L}$ phosphate buffered saline except in the case of lba-1 where Tris-buffered saline (TBS) was used. Antigen retrieval was done by heating the slides in citrate buffer (pH 6.0 ) at near boiling temperature $\left(95^{\circ} \mathrm{C}\right)$ in a water bath for 20 minutes. Quenching of endogenous peroxidase was done by treating with $1 \% \mathrm{H}_{2} \mathrm{O}_{2}$ in methanol for 30 min. After washing, blocking was done with appropriate $5 \%$ serums prepared in $0.1 \mathrm{~m}$ PBS. The slides were then incubated with respective primary or secondary antibodiesdiluted in $2.5 \%$ serums,each overnight, at $4^{0} \mathrm{C}$. PBS washed slides were then incubated with ExtrAvidin $\AA$ peroxidase (Sigma-Aldrich Pty. Ltd) for 3h at room temperature. Finally sections were treated with SIGMA FAST ( ${ }^{\mathrm{TM}}$ ) 3, 3'- diaminobenzidine tetrahydrochoride (Sigma-Aldrich Pty. Ltd). Negative control sections were processed in the same way except that primary antibody was excluded from the incubating solution. Caspse-3 immunolabelled slides were counterstained with weak thionin solution to distinguish the area for counting.Alcohol dehydrated sections were then cleared in xylene and permanently mounted with DPX.

All immunohistochemical assessments were done in a masked fashion in both ipsilateral and contralateral hemispheres. Neuronal survival was assessed by estimating NeuN positive cells in the lateral cortex, CA1-2, CA3, CA4 sub regions of the hippocampus, dentate gyrus and the striatum. Iba-1, TNF-a, GFAP and Caspase-3 positive cells were estimated in cortex and hippocampus. Immunopositive cell counts were done using Stereo Investigator software (Ver. 10, MBF Bio Science, Vermont, USA) by light microscopy (Nikon eclipse 80i, Tokyo, Japan). A contour was drawn at $2 x$ magnification covering the region of interest. The cells were counted after random sampling by Stereo Investigator at $40 x$ magnification by applying a fractionator probe consisting of a counting frame for object inclusion/exclusion. The grid and counting frame sizes applied for cortex and striatum were $400 \times 400 \mu \mathrm{m}$ and $100 \times 100 \mu \mathrm{m}$ respectively and for hippocampal sub regions were $100 \times 100 \mu \mathrm{m}$ and $75 \times 75 \mu \mathrm{m}$. Cells touching the bottom and left redlines of the counting frame were excluded whereas those touching the top and right green lines were included. Immunopositive cells thus estimated by the Stereo Investigator software were converted to cell density (cells/mm ${ }^{2}$ ) by applying the equation: Estimated number of immunopositive cells/ region of interest $\left.\left(\mu \mathrm{m}^{2}\right)\right] \times 10^{6}$.

\section{Statistics:}

The treatment effects of G-2MePE on the tissue damage scores (thionin/acid fuchsine), neuronal survival (NeuN), immunomodulation (lba-1, GFAP, TNF-a) and apoptosis (Caspase-3) on the ipsilateral and contralateral sides of the brain were analysed using two way ANOVA followed by Bonferroni post-hoc tests, with brain regions and treatments as dependant factors. Data are presented as mean \pm SEM. Statistical significance was assumed at $\mathrm{P}<0.05$.

Results G-2MePE treatment attenuated brain tissue damage after $\mathrm{HI}$ injury 
$\mathrm{HI}$ induced unilateral damage in the middle cerebral artery territoryipsilateral to the ligated carotid arteries including the lateral cortex (Figure $1 \mathrm{~A}$ ), hippocampus (Figure $1 \mathrm{C}, \mathrm{E} \mathrm{G}$ ) and striatum (Figure 1I). The insult was associated with selective neuronal loss, acidophilic staining in cytoplasm, tissue pan-necrosis, cellular reactions and tissue infarction.
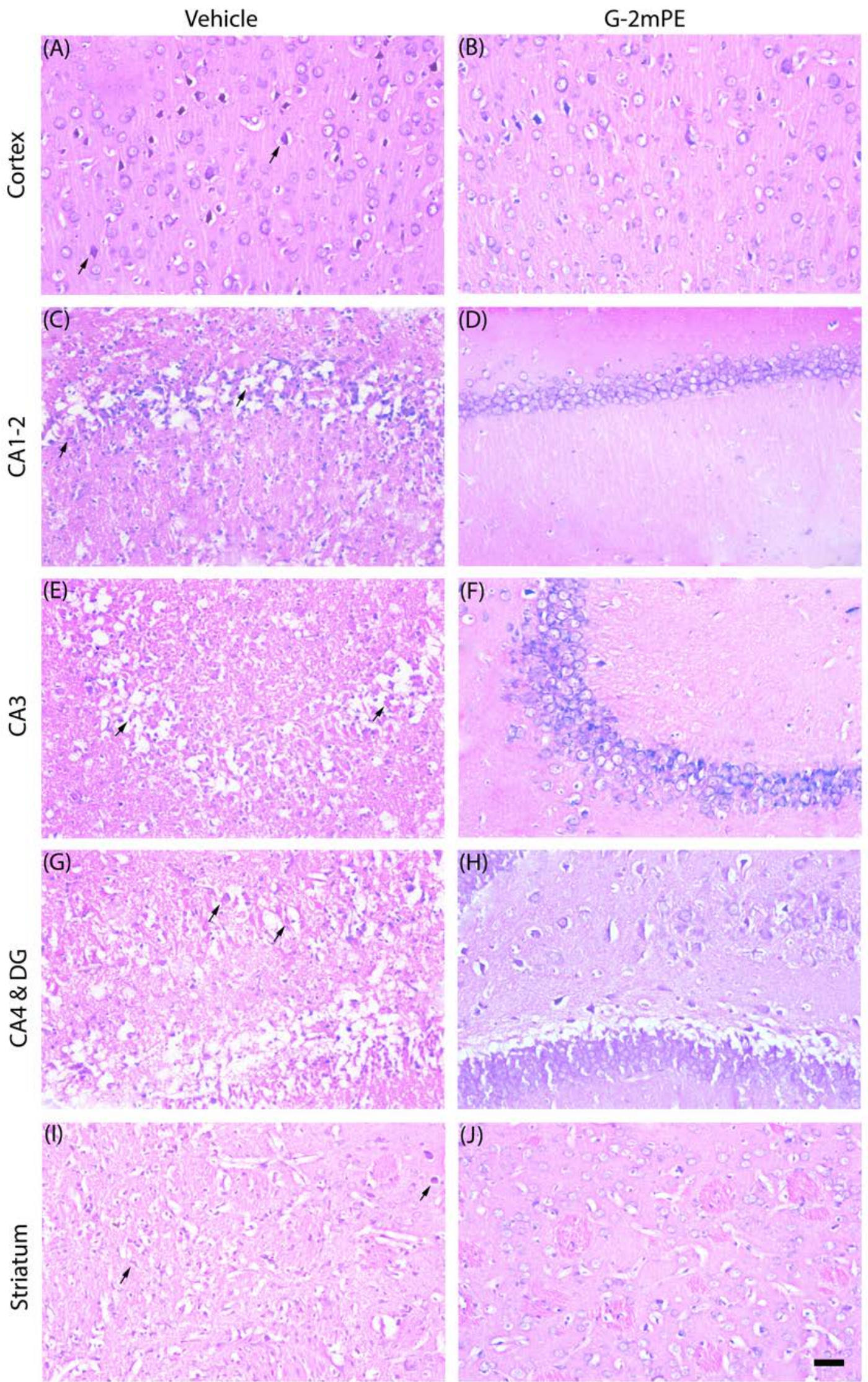
Figure 1.

Photomicrographs showing hypoxic-ischemic injury in different areas of rat brain after vehicle or G-2MePE treatment. Brains tissues extracted 5 days post $\mathrm{HI}$, formalin fixed, coronally cut at $8 \mu \mathrm{m}$ thickness and stained with thionin and acid fuchsin. Left panel shows sections from vehicle treated brains (A, C, E, G, I) and right panel shows brain sections from $\mathrm{G}-2 \mathrm{MePE}(1.2 \mu \mathrm{g} / \mathrm{kg})$ sub-cutaneously treated rats $3 \mathrm{~h}$ after $\mathrm{HI}$ injury $(\mathrm{B}, \mathrm{D}, \mathrm{F}, \mathrm{H}, \mathrm{J})$. Note the lesser number of dead or damaged neurons in the $\mathrm{G}-$ $2 \mathrm{MePE}$ treated group. Arrowheads represent areas of pan-necrosis. Neurons undergoing ischemic cell changes were characterised by pink acidophilic cytoplasm and contracted nuclei as shown by arrows. CA, cornu ammonis of hippocampus; DG, dentate gyrus. Scale bar $=40 \mu \mathrm{m}$

Sub cutaneous treatment with $1.2 \mathrm{mg} / \mathrm{kg} \mathrm{G}-2 \mathrm{MePE}$ reduced overall tissue damage scores compared to that of vehicle treated animals (F [1,156] $=15.85, p=0.05$, Figure 2A). However, post-hoc tests did not show specific protective effects of G-2MePE in the individual brain regions.

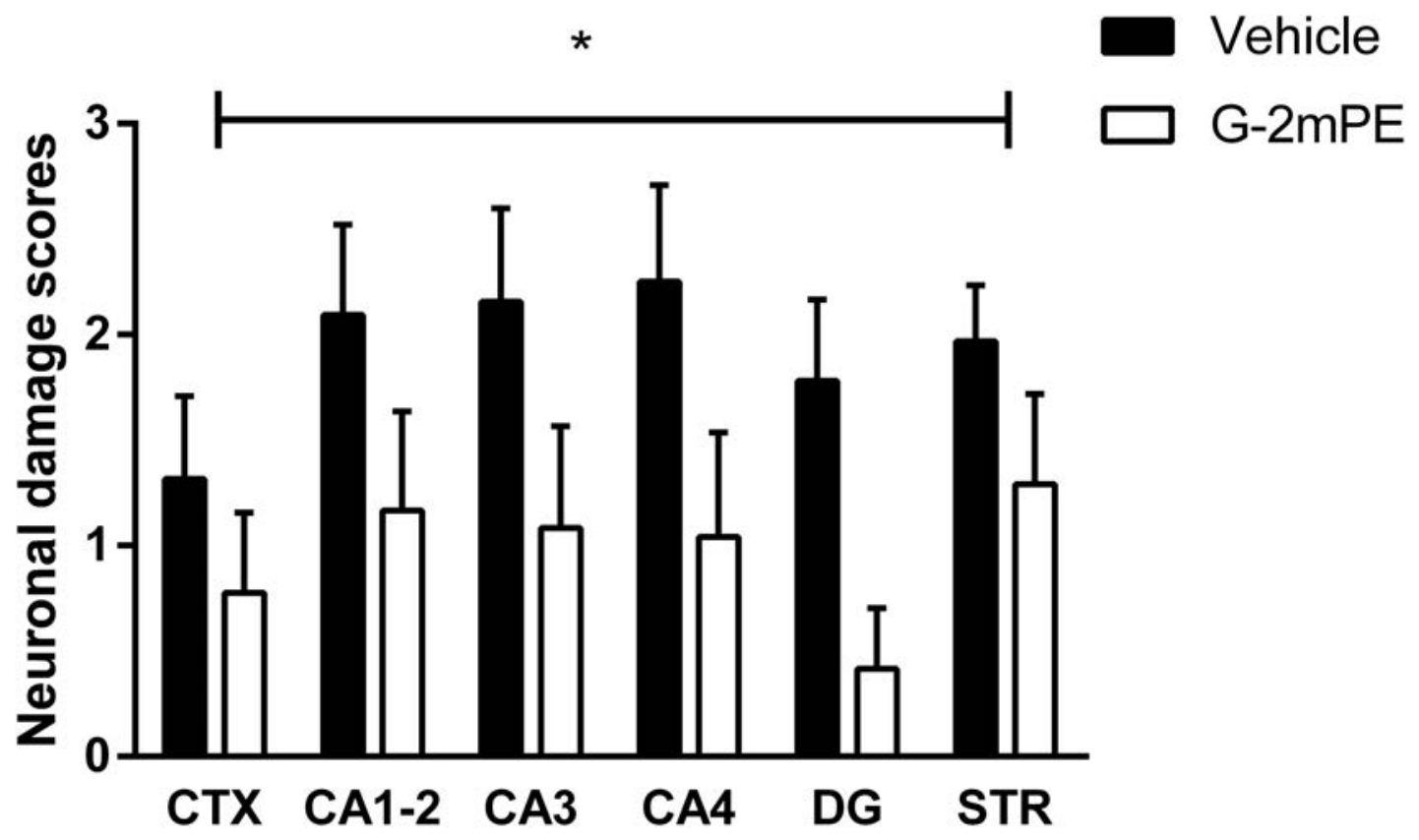

Figure 2.

Hypoxic ischemic Brain damage scores after treatment with vehicle or G-2MePE on brain regions. Sub-cutaneous treatment with G-2MePE $(1.2 \mathrm{mg} / \mathrm{kg}) 3 \mathrm{~h}$ post hypoxic-ischemic brain injury caused an overall reduction in brain damage scores Data are presented as mean \pm SEM * $p<0.05 ;{ }^{* *} p$

\section{G-2MePE treatment improved neuronal survival}

Photomicrographs show the distribution and morphological changes of neuronal survival in experiment 1 (Figure $3 \mathrm{~A}-\mathrm{J}$ ). Morphologically, the surviving neurons in the injured areas were smaller in size, with fewer processes and dendrites. Compared to the vehicle treated group, G-2MePE treated neurons showed almost normal neuronal morphology in all brain regions. There was no difference in number of neurons in the vehicle treated and G-2MePE treated experimental groups in the contralateral cortex, CA1-2, CA3, CA4, dentate gyrus or striatum (Figure 4A-F). HI injury caused significant loss of NeuN positive neurons in the ipsilateral cortex, sub regions of hippocampus and the striatum, with more severe loss in the cortex $(p<0.001), C A 3, C A 4$ areas of the hippocampus $(p<0.001)$ and striatum $(p<0.001)$. Sub cutaneous treatment with G-2MePE significantly improved the neuronal density (cells $/ \mathrm{mm}^{2}$ ) associated with $\mathrm{HI}$ injury on the ipsilateral cortex $(598.75 \pm 93.45$ vs $882.52 \pm 60.52 ; \mathrm{p}<0.05)$, CA1-2 $(975.89 \pm 245.40$ vs $1733.36 \pm 112.77$; $p<0.01)$, CA3 (371.55 \pm 78.62 vs $1082.64 \pm 134.36$; $p<0.001)$, CA4 (229.38 \pm 39.34 vs $414.57 \pm 17.67$; $\mathrm{p}<0.001)$ areas of the hippocampus, the dentate gyrus $(1229.52 \pm 309.56$ vs $2897.94 \pm 155.04 ; p<0.001)$ and striatum $(544.22 \pm 109.23$ vs 1023.74 \pm 50.23 ; $p<0.001$ ) to a level similar to the counts in contra lateral counterpart area (Figure 4A-F). 

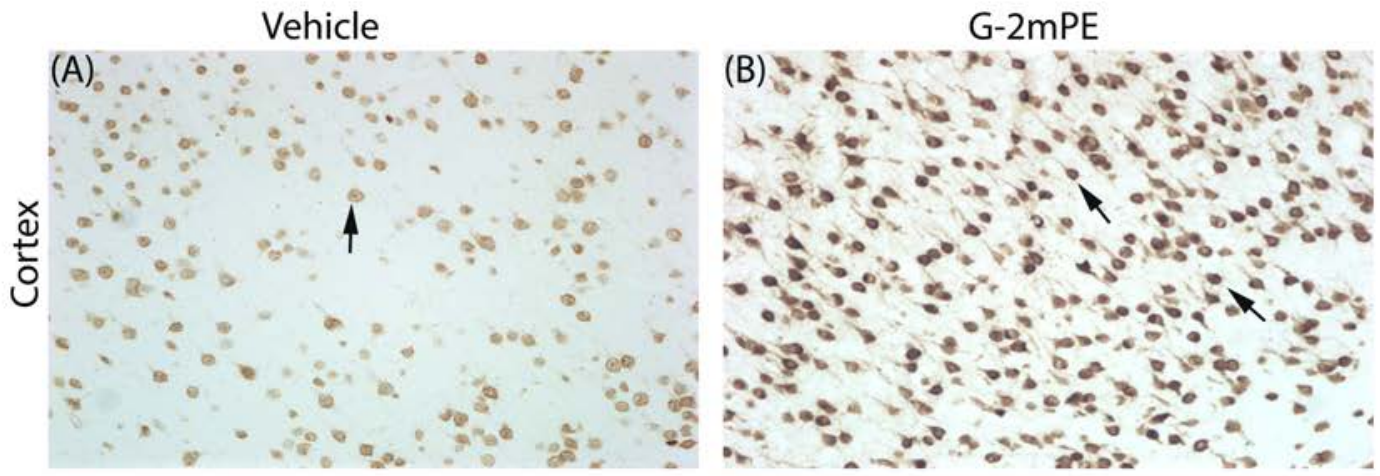

(C)
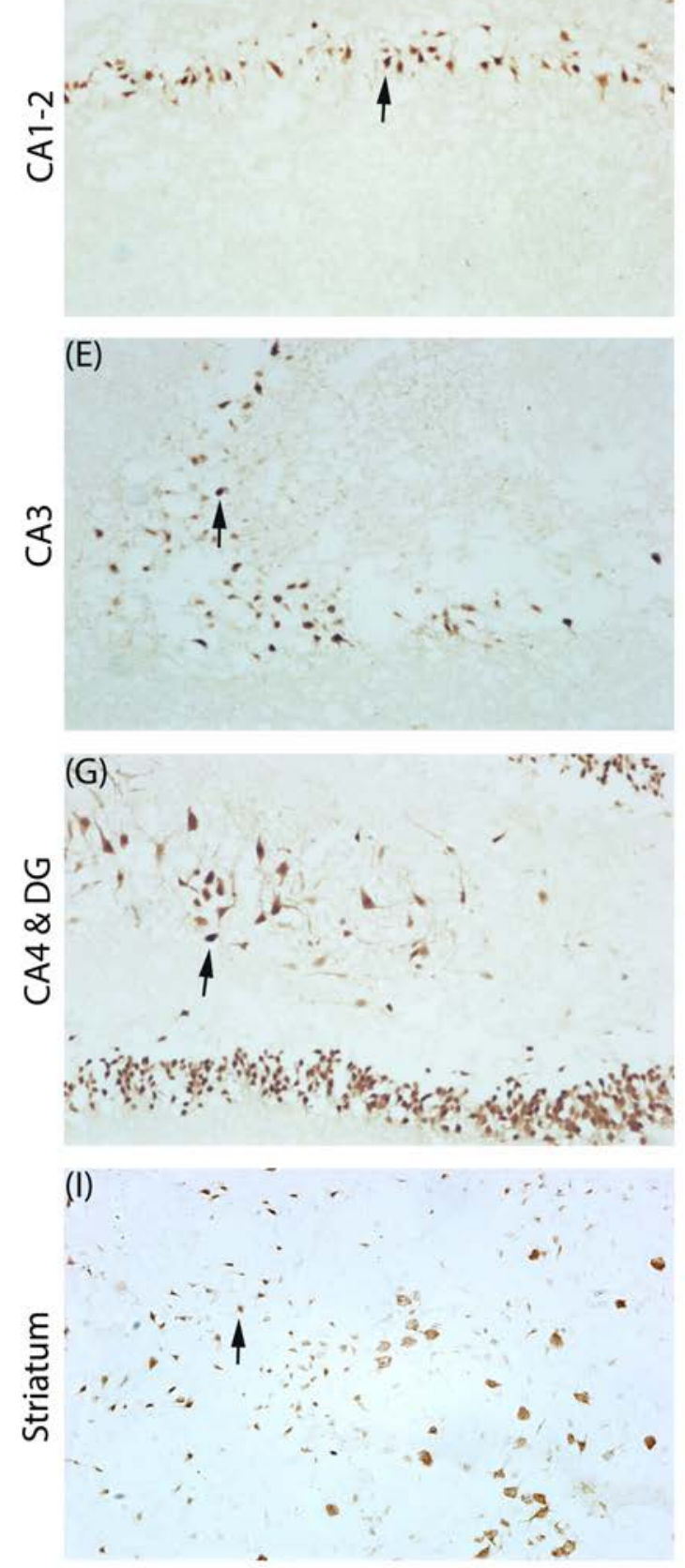

(D)
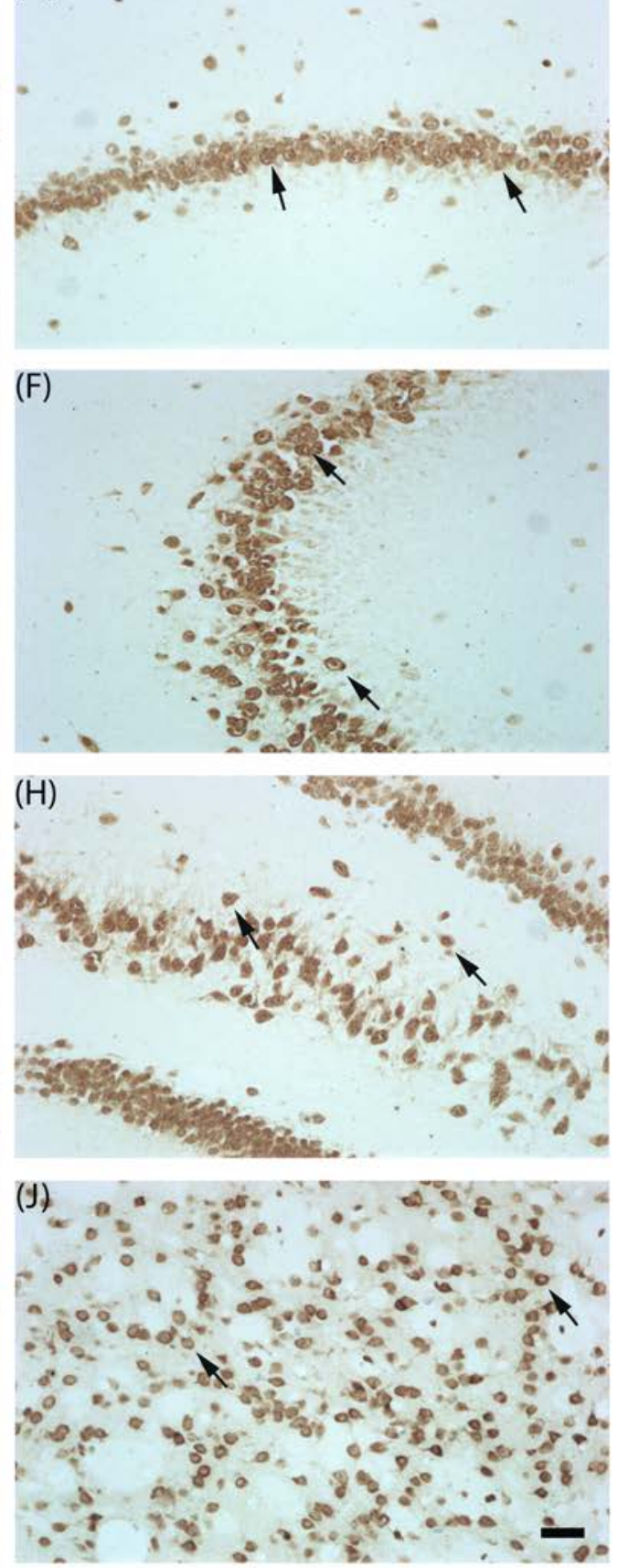

Figure 3.

Representative photomicrographs showing morphology of NeuN immunopositve neurons in HI brain regions after vehicle or G- 
2MePE treatment. $\mathrm{HI}$ injury caused apparent reduction in the neuronal population and many cells appeared pycnotic(arrow)

whereas subcutaneous treatment with G-2MePE $(1.2 \mathrm{mg} / \mathrm{kg})$ was associated with improved population in cortex (A, B), CA1-

$2(C, D), C A-3(G, H), C A 4$ areas of the hippocampus, the dentate gyrus $(G, H)$ and the striatum $(I, J)$ and the cells appeared morphologically normal. Scale bar $=40 \mu \mathrm{m}$.

(A)

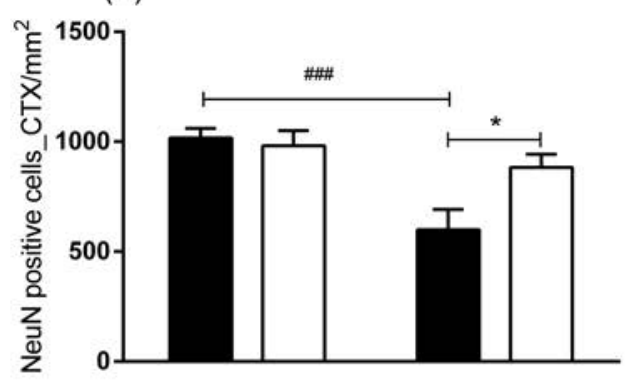

(B)

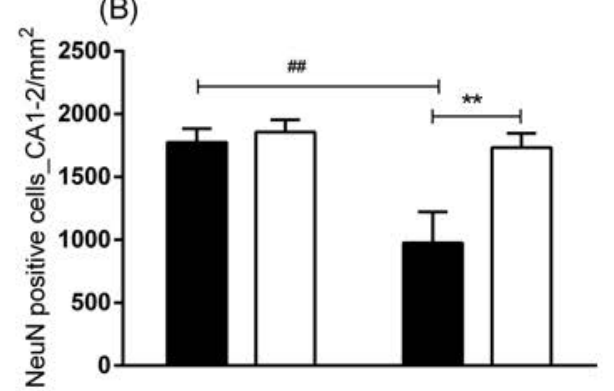

(C)

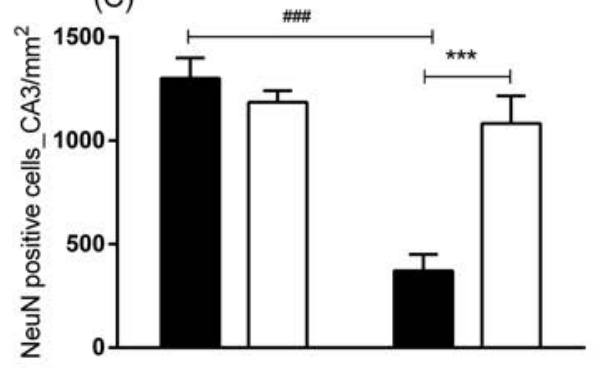

(D)

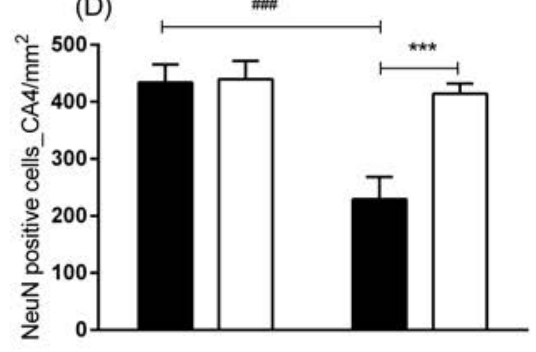

(E)

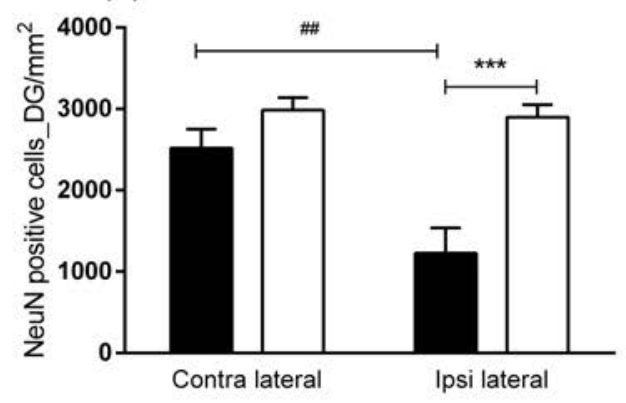

(F)

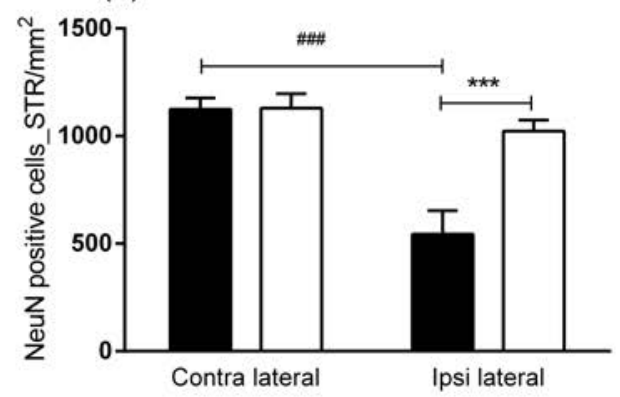

Figure 4.

Neuronal counts (cells/ mm2) in HI brain regions after vehicle or G-2MePE treatment. HI injury caused a remarkable decrease in the neuronal density in the ipsilateral side of the brain in the cortex (A), CA1-2 (B), CA3 (C), CA-4 areas of the hippocampus, the dentate gyrus $(E)$ and striatum $(F)$. Sub-cutaneous treatment with $\mathrm{G}-2 \mathrm{MEPE}(1.2 \mathrm{mg} / \mathrm{kg}) 3 \mathrm{~h}$ post $\mathrm{HI}$ was associated with a 

significant improvement in the neuronal outcome in all these brain areas analysed. Data are presented as mean \pm SEM. \#\#p

\section{G-2MePE SC treatment augmented astrocytosis}

Astrocytes were present throughout hippocampus and cortex. $\mathrm{HI}$ injury was associated with induction of hypertrophic astrocytes with thicker cell bodies (Figure 5A, B). GFAP positive cell counts (cells $/ \mathrm{mm}^{2}$ ) in the hippocampus and cortex were similar between the two hemispheres of the vehicle treated group hippocampus and cortex. Treatment with G-2MePE was associated with a significant increase in reactive astrocytes in the ipsilateral hippocampus (184.52 \pm 29.19 vs $302.79 \pm 47.73, p<0.05)$ and cortex $(144.04 \pm 23.78$ vs $291.18 \pm 42 ; p<0.001 ;(F i g u r e 6 A$, B).
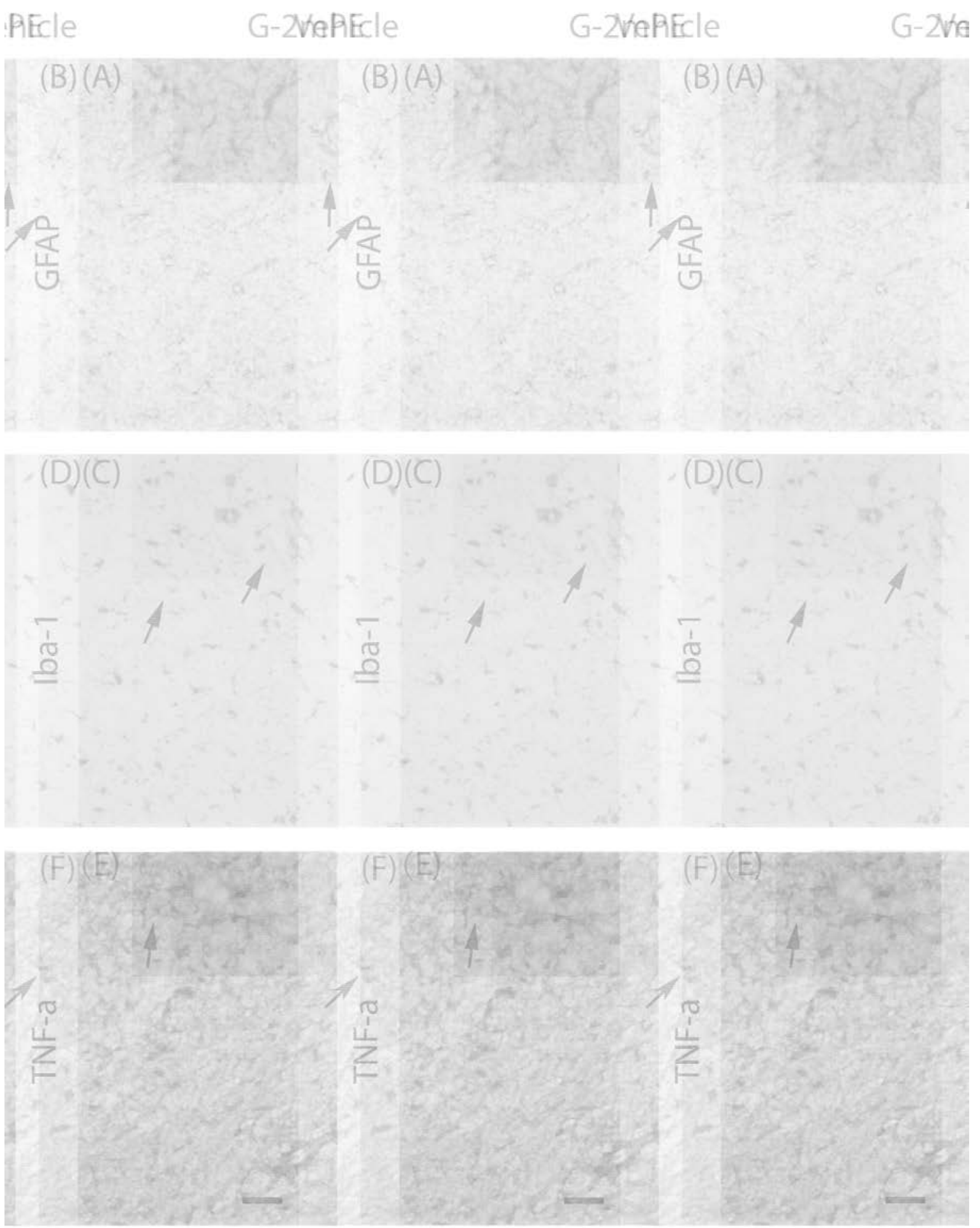

Figure 5.

Photomicrographs showing astrocytes, microglia, TNFa positive cells, and cleaved caspase- 3 positive cells in $\mathrm{HI}$ cortex and hippocampus after vehicle or G-2MePE treatment. HI injury was associated with remarkable induction of astrocytes in the vehicle ipsilateral cortex $(A)$ while G-2MePE treatment was associated with a further increase of hypertrophic astrocytes in the 
treated ipsilateral cortex (B). Also, G-2MePE treatment was associated with marked attenuation of inflammatory responses as

evidenced by reduced number of activated microglia (D), TNFa positive cells (F) \& reduced number of caspase-3 positive cells

in the ipsilateral treatedcortex $(\mathrm{H})$. Corner inserts are the images of cells (arrow marked) magnified. Scale bar A-F=40 4 m;

$\mathrm{GH}=20 \mu \mathrm{m}$.

(A)

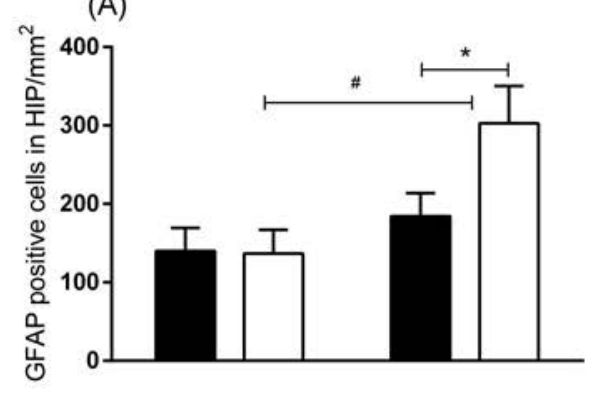

(C)

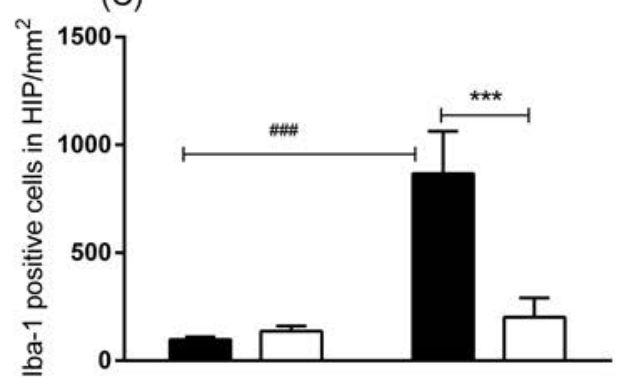

(E)

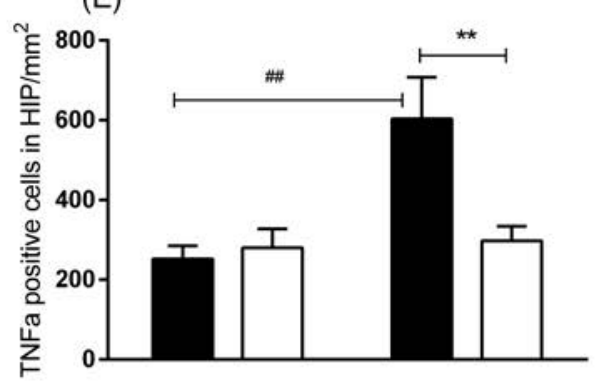

(B)
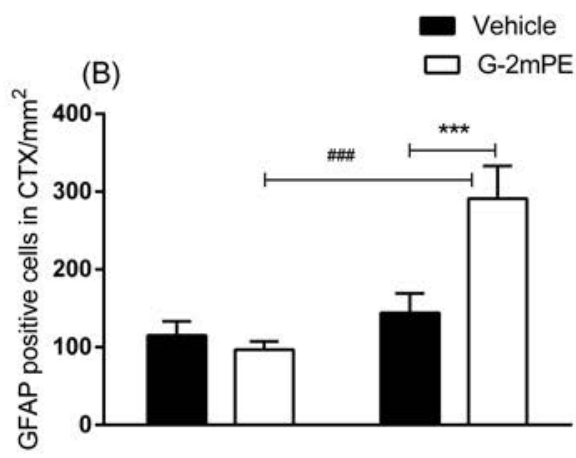

(D)

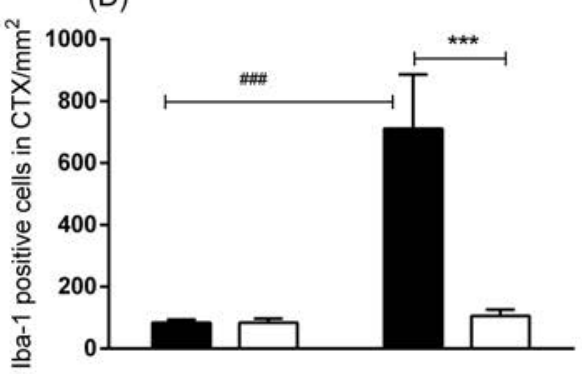

$(\mathrm{F})$

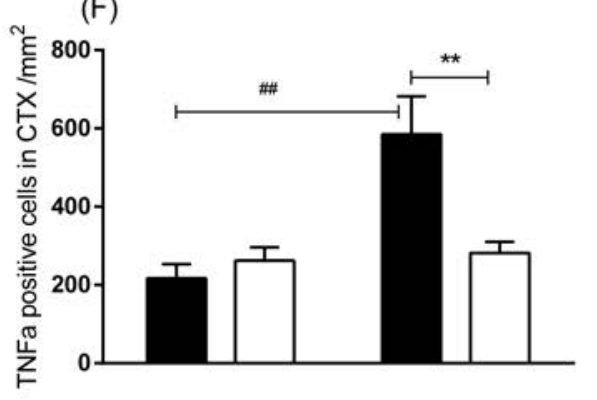


Figure 6.

Astrocytes, microglia, TNFa positive and caspase -3 positive cell counts in HI brain hippocampus and cortex after vehicle or G$2 \mathrm{MePE}$ treatment. There was a significant increase in GFAP positive astrocytes in the ipsilateral hippocampus and cortex (A, B) after G2-mPE treatment. On the contrary sub-cutaneous treatment with G-2MePE was associated with a marked decrease in the absolute numbers in reactive microglia(C, D), TNF- $\alpha$ positive cells $(E, F)$ and caspse-3 positive cells $(G, H)$ in the ipsilateral hippocampus and cortex. HIP= Hippocampus, CTX= cortex. Data are mean \pm SEM. \#\#p

\section{G-2MePE treatment inhibited microglial activation}

$\mathrm{HI}$ injury was associated with marked increase in reactive microglia with amoeboid morphology, throughout the ipsilateral hemisphere. There was intense infiltration in the necrotic areas (Figure 5C). Compared to the contralateral hemisphere there was approximately a 9 fold increase in reactive microglia population in the ipsilateral hippocampus and 8.5 fold increase in the lateral cortex. Treatment with G-2MePE significantly reduced the density of microglia (cells $/ \mathrm{mm}^{2}$ ) in the ipsilateral hippocampus $(867.08 \pm 195.49$ vs 201.37 \pm 88.98 ; p $<0.001)$ and cortex (711.41 \pm 174.80 vs 105.65 $\pm 20.24 ; p<0.001$; Figure 6C, D).

\section{G-2MePE treatment inhibited TNF- $\alpha$ expression}

$\mathrm{HI}$ injury caused infiltration and release of pro-inflammatory cytokines. TNF- $\alpha$ positive cells were diffusely distributed in all sub-regions of the hippocampus and the cortex (Figure 5E, F). HI injury was associated with a 2.4 fold increase in TNF- $\alpha$ positive cells in the ipsilateral hippocampus and 2.7 fold increase in cortex. Treatment with G-2MePE significantly reduced TNF- $\alpha$ positive cell counts (cells/mm ${ }^{2}$ ) in the ipsilateral hippocampus $(603.5 \pm 104$ vs $297.71 \pm 36.5 p<0.01)$ and cortex $(585.3 \pm 96.7$ vs $281.4 \pm 28.95 ; p<0.01)$, almost to the vehicle control levels (Figure $6 \mathrm{E}, \mathrm{F})$.

\section{G-2MePE treatment reduced caspase -3 mediated apoptosis}

Hypoxic-ischemic injury was associated with induction of caspase-3 mediated apoptotic cells in the ipsilateral side of the hippocampus and cortex. Morphologically there was a gradient of caspase-3 labelled cells; most recently cleaved caspase being densely stained (Figure $5 \mathrm{G}$, $\mathrm{H}$ ). $\mathrm{G}-2 \mathrm{MePE}$ treatment significantly reduced the apoptotic cell counts (cells $/ \mathrm{mm}^{2}$ ) in the ipsilateral hippocampus $(132.63 \pm 17.72$ vs $68.62 \pm 11.22 ; \mathrm{p}<0.01)$ and cortex (86.97 \pm 12.54 vs $53.60 \pm 8.02 ; \mathrm{p}<0.05$; Figure $6 \mathrm{G}, \mathrm{H})$.

\section{Discussion}

In the present study G-2MePE treatment, by a single dose SC administration significantly reduced the extent of brain damage after HI injury. Attenuation of brain damage, neuronal loss and apoptosis were associated with reduced microglial reaction, TNF- $\alpha$ expression and greater astrocytosis in G-2MePE treated group.

The modified analogue of GPE, G-2MePE, has a much longer half-life in plasma (49 min) compared to GPE (2 min) due to improved enzymatic resistance (Bickerdike et al. , 2009). Although ANOVA indicated an overall reduction in tissue damage scores after single dose treatment the efficacy in individual brain regions was not significant. The treatment effects of G-2MePE were further also evaluated by measuring the number of survival neurons using NeuN immunolabelling. We found the significant improvements of neuronal survival in all brain regions examined. While the tissue damage scores reflected the changes that associated with necrotic neuronal death the evaluation of surviving neurons appeared to be a more sensitive measure for the treatment effects of G-2MePE. In addition, HI injury also induced obvious morphological changes in the neurons by showing the deformed and shrinking cell bodies. The treatment of G-2MePE also prevented HI-induced morphological changes in neurons.

The protective effects of G-2MePE were also demonstrated by showing reduced apoptotic neuronal death. It is known that the activation of caspase -3 is involved in neuronal death after hypoxic-ischemia particularly in brain regions with more progressive neuronal loss (Guan et al. , 2013). There were $50 \%$ more caspase- 3 positive cells in the ipsilateral hippocampus than on the ipsilateral cortex, suggesting neuronal death may be more progressive in the hippocampus whereas necrotic death may be the major factor involved in cortical neuronal loss. Thus the protective effects of G-2MePE may be mediated through necrotic and apoptotic pathways.

Unilateral ligation of the carotid artery induces a relative ischemia without causing any tissue damage due to collateral blood supply from the contralateral hemisphere. The ischemia associated brain injury in the brain regions of middle cerebral artery territory occurs only when the collateral circulation is damaged, in this case the brief hypoxic insult. Unlike the ischemic-reperfusion injury from either a transient or permanent middle cerebral artery occlusion, the brain damage induced by $\mathrm{HI}$ appears to be more progressive and possibly more reversible. Consistent with the present study Bickerdike and colleagues (2009) reported that continuous intravenous infusion of G-2MePE reduces the infarct size after endothelin1 (ET1) induced middle cerebral artery vasoconstriction in rats (Bickerdike et al. , 2009). Zhao et al (2005) demonstrated that G-2MePE offers effective neuroprotection by reducing neuronal deficit scores at a 10 times lower dose compared to GPE, when treated in combination with caffeinol (Zhao et al. , 2005).

Microglia plays an important role in the inflammatory response in the brain and produce pro-inflammatory cytokines like TNF- $\alpha$ or IL-1 $\beta$. We observed inhibition of reactive microglia and expression of TNF- $\alpha$ after G-2MePE treatment. This is consistent with evidence that anti-inflammation contributes to the neuroprotection of G-2MePE as the improved neuronal outcome was associated with reduction of pro-inflammatory cytokine IL-6 after $\mathrm{HI}$ injury in neonatal rats (Svedin et al. , 2007). The effects of TNF- $\alpha$ are mediated through two membrane receptors, TNF- $\alpha$-receptor 1 \&2, and activation of one of these receptors lead to cell death (Aggarwal and Natarajan, 1996, Nakazawa et al. , 2006).

The SC treatment with G-2MePE also induced a marked increase in reactive astrocytes in the ipsilateral brain after HI. Reactive astrocytes play a critical role in protecting neurons, maintaining blood-brain barrier integrity, cell-to-cell communication and neuronal plasticity (Kraig et al. , 1995). We have previously reported loss of astrocytes in the CA1-2 and CA4 sub-regions of the hippocampus after HI in adult rats and that treatment with GPE prevented the loss of astrocytes. This may be apparently associated with the increase in astrocytes in the present study (Guan, 2008). The effects of GPE on astrocytosis appeared to be brain region specific as there was no change in CA3 and the cortex. Astrocytes also have been implicated in the neo-vascularisation process (Salhia et al. , 2000, Acker et al. , 2001). Given a role for astrocytes in vascular remodelling, the astrocytosis may also be associated with the vascular protection and/or remodelling after GPE (Shapira et al. , 2009) and G-2MePE treatment in neonatal rats (Svedin et al. , 2007).

Given that GPE, presumably the analogue, does not interact with IGF-1 receptors the mechanism of neuroprotective effects of GPE has been recently suggested to be mediated through improving bioavailability of IGF-1 (Guan et al. , 2014), which needs to be further investigated in future study. 
G-2MePE is neuroprotective in adult rats when administered $3 \mathrm{~h}$ after $\mathrm{HI}$ injury sub-cutaneously. The mechanisms of neuroprotection may include blocking the necrotic or apoptotic pathways of cell death as well as modulating the immune response and augmenting astrocytosis.

\section{References}

Acampa, M, Guideri, F, Tassi, R, D’Andrea, P, Marotta, G, Giudice, GL ,Martini, G (2014). Thrombolytic Treatment of Cardiac Myxoma-Induced Ischemic Stroke: a Review. Curr Drug Saf.

Acker, T, Beck, H ,Plate, $\mathrm{KH}$ (2001). Cell type specific expression of vascular endothelial growth factor and angiopoietin-1 and -2 suggests an important role of astrocytes in cerebellar vascularization. Mech Dev 108: 45-57.

Aggarwal, BB ,Natarajan, K (1996). Tumor necrosis factors: developments during the last decade. Eur Cytokine Netw 7: 93-124.

Alonso De Diego, SA, Gutierrez-Rodriguez, M, Perez de Vega, MJ, Casabona, D, Cativiela, C, Gonzalez-Muniz, R, Herranz, R, Cenarruzabeitia, E, Frechilla, D, Del Rio, J, Jimeno, ML ,Garcia-Lopez, MT (2006). New Gly-Pro-Glu (GPE) analogues: expedite solid-phase synthesis and biological activity. Bioorg Med Chem Lett 16: 1392-6.

Auer, RN, Kalimo, H, Olsson, Y,Siesjo, BK (1985). The temporal evolution of hypoglycemic brain damage. II. Light- and electron-microscopic findings in the hippocampal gyrus and subiculum of the rat. Acta Neuropathol 67: 25-36.

Batchelor, DC, Lin, H, Wen, JY, Keven, C, Van Zijl, PL, Breier, BH, Gluckman, PD ,Thomas, GB (2003). Pharmacokinetics of glycine-prolineglutamate, the $\mathrm{N}$-terminal tripeptide of insulin-like growth factor-1, in rats. Anal Biochem 323: 156-63.

Bickerdike, MJ, Thomas, GB, Batchelor, DC, Sirimanne, ES, Leong, W, Lin, H, Sieg, F, Wen, J, Brimble, MA, Harris, PW ,Gluckman, PD (2009). NNZ-2566: A Gly-Pro-Glu analogue with neuroprotective efficacy in a rat model of acute focal stroke. J Neurol Sci 278: 85-90.

Brown, AW ,Brierley, JB (1972). Anoxic-ischaemic cell change in rat brain light microscopic and fine-structural observations. J Neurol Sci 16: 5984.

Cartagena, CM, Phillips, KL, Williams, GL, Konopko, M, Tortella, FC, Dave, JR ,Schmid, KE (2013). Mechanism of action for NNZ-2566 antiinflammatory effects following PBBI involves upregulation of immunomodulator ATF3. Neuromolecular Med 15: 504-14.

Chan, $\mathrm{PH}$ (2001). Reactive oxygen radicals in signaling and damage in the ischemic brain. J Cereb Blood Flow Metab 21: 2-14.

Dirnagl, U, ladecola, C ,Moskowitz, MA (1999). Pathobiology of ischaemic stroke: an integrated view. Trends Neurosci 22: $391-7$.

Domanska-Janik, K, Buzanska, L, Dluzniewska, J, Kozlowska, H, Sarnowska, A ,Zablocka, B (2004). Neuroprotection by cyclosporin A following transient brain ischemia correlates with the inhibition of the early efflux of cytochrome C to cytoplasm. Brain Res Mol Brain Res 121: $50-9$.

Fisher, M ,Schaebitz, W (2000). An overview of acute stroke therapy: past, present, and future. Arch Intern Med 160: $3196-206$

Gheibi, S, Aboutaleb, N, Khaksari, M, Kalalian-Moghaddam, H, Vakili, A, Asadi, Y, Mehrjerdi, FZ,Gheibi, A (2014). Hydrogen Sulfide Protects the Brain Against Ischemic Reperfusion Injury in a Transient Model of Focal Cerebral Ischemia. J Mol Neurosci. 54: 264-70

Gleichmann, M, Weller, M ,Schulz, JB (2000). Insulin-like growth factor-1-mediated protection from neuronal apoptosis is linked to phosphorylation of the pro-apoptotic protein BAD but not to inhibition of cytochrome c translocation in rat cerebellar neurons. Neurosci Lett 282 : $69-72$.

Guan, J (2008). Insulin-like growth factor-1 and its derivatives: potential pharmaceutical application for ischemic brain injury. Recent Pat CNS Drug Discov 3: 112-27.

Guan, J, Gluckman, P, Yang, P, Krissansen, G, Sun, X, Zhou, Y, Wen, J, Phillips, G, Shorten, PR, McMahon, CD, Wake, GC, Chan, WHK, Thomas MF, Ren, A, Moon, S ,Liu, D-X (2014). Cyclic glycine-proline regulates IGF-1 homeostasis by altering the binding of IGFBP-3 to IGF-1. Sci. Rep. 4.

Guan, J, Gunn, AJ, Sirimanne, ES, Tuffin, J, Gunning, MI, Clark, R, Gluckman, PD (2000). The window of opportunity for neuronal rescue with insulin-like growth factor-1 after hypoxia-ischemia in rats is critically modulated by cerebral temperature during recovery. $\mathrm{J}$ Cereb Blood Flow Metab 20: 513-9.

Guan, J, Mathai, S, Liang, HP ,Gunn, AJ (2013). Insulin-like growth factor-1 and its derivatives: potential pharmaceutical application for treating neurological conditions. Recent Pat CNS Drug Discov 8: 142-60.

Guan, J, Thomas, GB, Lin, H, Mathai, S, Bachelor, DC, George, S ,Gluckman, PD (2004). Neuroprotective effects of the N-terminal tripeptide of insulin-like growth factor-1, glycine-proline-glutamate (GPE) following intravenous infusion in hypoxic-ischemic adult rats. Neuropharmacology 47: 892-903.

Guan, J, Williams, C, Gunning, M, Mallard, C ,Gluckman, P (1993). The effects of IGF-1 treatment after hypoxic-ischemic brain injury in adult rats. J Cereb Blood Flow Metab 13: 609-16.

Harris, PW ,Brimble, MA (2006). Synthesis of macrocyclic analogues of the neuroprotective agent glycyl-L-prolyl-L-glutamic acid (GPE). Org Biomol Chem 4: 2696-709.

Hayashi, T ,Abe, K (2004). Ischemic neuronal cell death and organellae damage. Neurol Res 26: 827-34.

Kawano, T, Fukunaga, K, Takeuchi, Y, Morioka, M, Yano, S, Hamada, J, Ushio, Y ,Miyamoto, E (2001). Neuroprotective effect of sodium orthovanadate on delayed neuronal death after transient forebrain ischemia in gerbil hippocampus. J Cereb Blood Flow Metab 21: 1268-80.

Kilic, E, Kilic, U ,Hermann, DM (2006). TAT fusion proteins against ischemic stroke: current status and future perspectives. Front Biosci 11: 171621.

Kraig, RP, Lascola, CD ,Caggiano, A (1995). Glial response to brain ischemia. Neuroglia 64: 964-76. 
Lu, XC, Chen, RW, Yao, C, Wei, H, Yang, X, Liao, Z, Dave, JR , Tortella, FC (2009). NNZ-2566, a glypromate analog, improves functional recovery and attenuates apoptosis and inflammation in a rat model of penetrating ballistic-type brain injury. J Neurotrauma 26: $141-54$.

Mohammad, YM, Divani, AA, Kirmani, JF, Harris-Lane, P ,Qureshi, Al (2004). Acute treatment for ischemic stroke in 2004. Emerg Radiol 11: 83-6.

Nakazawa, T, Nakazawa, C, Matsubara, A, Noda, K, Hisatomi, T, She, H, Michaud, N, Hafezi-Moghadam, A, Miller, JW ,Benowitz, LI (2006). Tumor necrosis factor-alpha mediates oligodendrocyte death and delayed retinal ganglion cell loss in a mouse model of glaucoma. J Neurosci 26 : 12633-41.

Paxinos, G,Watson, CD (1982). The rat brain in sterotaxic coordinations. New York, Academic press.

Rochester, MA, Riedemann, J, Hellawell, GO, Brewster, SF ,Macaulay, VM (2005). Silencing of the IGF1R gene enhances sensitivity to DNAdamaging agents in both PTEN wild-type and mutant human prostate cancer. Cancer Gene Ther 12: 90-100.

Salhia, B, Angelov, L, Roncari, L, Wu, X, Shannon, P, Guha, A, Giulian, D, Woodward, J, Young, DG, Krebs, JF ,Lachman, LB (2000). Expression of vascular endothelial growth factor by reactive astrocytes and associated neoangiogenesis Brain Res 883: 87-97.

Sara, VR, Carlsson-Skwirut, C, Bergman, T, Jornvall, H, Roberts, PJ, Crawford, M, Hakansson, LN, Civalero, I ,Nordberg, A (1989). Identification of Gly-Pro-Glu (GPE), the aminoterminal tripeptide of insulin-like growth factor 1 which is truncated in brain, as a novel neuroactive peptide. Biochem Biophys Res Commun 165: 766-71.

Shapira, S, Mathai, S, Zhang, R ,Guan, J (2009). Delayed peripheral administration of the N-terminal tripeptide of IGF-1 (GPE) reduces brain damage following microsphere induced embolic damage in young adult and aged rats. Neurosci Lett 454: 53-7.

Sizonenko, SV, Sirimanne, ES, Williams, CE ,Gluckman, PD (2001). Neuroprotective effects of the N-terminal tripeptide of IGF-1, glycine-prolineglutamate, in the immature rat brain after hypoxic-ischemic injury. Brain Res 922: 42-50.

Svedin, P, Guan, J, Mathai, S, Zhang, R, Wang, X, Gustavsson, M, Hagberg, H ,Mallard, C (2007). Delayed peripheral administration of a GPE analogue induces astrogliosis and angiogenesis and reduces inflammation and brain injury following hypoxia-ischemia in the neonatal rat. Dev Neurosci 29: 393-402.

Svedin, P, Guan, J, Mathai, S, Zhang, R, Wang, X, Gustavsson, M, Hagberg, H ,Mallard, C (2007). Delayed peripheral administration of a GPE analogue induces astrogliosis and angiogenesis and reduces inflammation and brain injury following hypoxia-ischemia in the neonatal rat. Dev Neurosci 29: 393-402.

Switzer, JA ,Hess, DC (2006). Statins in stroke: prevention, protection and recovery. Expert Rev Neurother 6: $195-202$.

Wahlgren, NG ,Ahmed, N (2004). Neuroprotection in cerebral ischaemia: facts and fancies-the need for new approaches. Cerebrovasc Dis 17 Suppl 1: 153-66.

Wei, HH, Lu, XC, Shear, DA, Waghray, A, Yao, C, Tortella, FC ,Dave, JR (2009). NNZ-2566 treatment inhibits neuroinflammation and proinflammatory cytokine expression induced by experimental penetrating ballistic-like brain injury in rats. J Neuroinflammation 6: 19 .

Zhao, X, Liu, SJ, Zhang, J, Strong, R, Aronowski, J ,Grotta, JC (2005). Combining insulin-like growth factor derivatives plus caffeinol produces robust neuroprotection after stroke in rats. Stroke 36: 129-34.

Zhou, R, Yang, Z, Tang, X, Tan, Y, Wu, X ,Liu, F (2013). Propofol protects against focal cerebral ischemia via inhibition of microglia-mediated proinflammatory cytokines in a rat model of experimental stroke. PLoS One 8: e82729.

\section{Acknowledgements}

Acknowledgements These studies were funded by the Health Research Council of New Zealand and Neuren Pharmaceuticals limited.Author ContributionsConceived and designed the experiments: JG. Performed the experiments: SM. Synthesis of the G-2MePE: PH MB. Analysis of the data: JG AG SM. Wrote the paper: SM JG AG 